\title{
Transgressive Segregation for Fibre Quality, Seed Cotton Yield and Its Component Traits in Gossypium barbadense L. Cotton
}

\author{
N.V. Mohan Kumar ${ }^{1 *}$, I.S. Katageri ${ }^{2}$, G. Sunil Kumar ${ }^{1}$ and S. Anjan Gowda ${ }^{1}$ \\ ${ }^{1}$ Department of Genetics and Plant Breeding, College of Agriculture, UAS, Dharwad-580005, \\ Karnataka, India \\ ${ }^{2}$ Associate Director of Research, RARS Vijayapura, UAS, Dharwad, Karnataka, India \\ *Corresponding author
}

A B S T R A C T

\begin{tabular}{|l|}
\hline Ke y w o r d s \\
$\begin{array}{l}\text { Transgressive } \\
\text { segregants, } \\
\text { Segregation, Seed } \\
\text { cotton and Fibre } \\
\text { strength }\end{array}$ \\
\hline Article Info \\
\hline $\begin{array}{l}\text { Accepted: } \\
\text { 10 December } 2017 \\
\text { Available Online: } \\
\text { 10 January } 2018\end{array}$ \\
\hline
\end{tabular}

\section{Introduction}

Cotton a king of fibre, known for its desirable properties is called 'White Gold' due to its global importance in industrial economy. In India, providing direct employment to around 35 million people. During 2015-16 India's export earnings from cotton yarn was 3.6 billion US \$, while export of cotton fabrics, cotton makeup's and raw cotton stood at 2.1, 5.2 and 1.9 billion US $\$$ respectively (Anon., 2016). Out of 50 Gossypium species only four have been cultivated worldwide, two diploid $(2 \mathrm{n}=2 \mathrm{x}=26)$ A genome species ( $G$. arborium and $G$. herbacium) and two $\mathrm{AD}$ genome $(2 \mathrm{n}=4 \mathrm{x}=52)$ species $(G$. hirsutum and $G$. barbadense). These cultivated species were independently getting domesticated for their commercial fibers (Wendel et al., 1999). Pima cotton or Egyptian cotton (Gossypium barbadense) is known for its finest fibre properties being cultivated in less than $1 \%$ in India (Anon, 2017), it is just because of its shy yielding ability, bad boll opening, higher susceptibility to sucking pests and boll worms. In India, long and extra-long staple cotton is widely grown in South Zone, Tamil Nadu, Andhra Pradesh and Karnataka as the climatic 
requirement for growing this cotton are more conducive and there has been a heavy demand for this cotton in the recent past in textile industry. This long and extra-long staple cotton varieties/hybrids are mainly based on Gossypium barbadense back ground. To meet industrial requirement there is an urgent need to improve yielding potential in Gossypium barbadense.

Seed cotton yield is a complex trait, it governed by several yield contributing characters such as plant height, number of monopodia, number of sympodia, number of bolls per plant and boll weight. In cotton, high seed cotton yield potential of Gossypium hirstum and superior fibre quality of Gossypium barbadense had been exploited simultaneously in the form of interspecific hybrids, Varalaxmi (first inter-specific hybrid developed by Katarki 1971), followed by DCH-32, NHB-12, HB-224 and TCHB-213 developed at various institutes.

However, due to the genetic deterioration of promising released varieties/hybrids, new genotypes with improved fibre properties are desirable to meet the demand in Indian textile industry. The genetic improvement of any crop is depends on the existence of initial genetic variability among the populations and how we are going to exploit it.

The best method for genetic variability create in sexually (seed) propagated crop is through wide hybridization or crossing between genetically diverse parents and can exploit these variation in the form of selecting transgressive segregants, intern develop a new variety or genetic stock for further improvement. Hence, present study was conducted to select transgressive segregants for seed cotton yield and fibre quality traits in segregating populations $\left(\mathrm{F}_{2}, \mathrm{~B}_{1}\right.$ and $\left.\mathrm{B}_{2}\right)$ of cross between Suvin and BCS 23-18-7 genotypes of Gossypium barbadense cotton.

\section{Materials and Methods}

The six generations $\left(\mathrm{P}_{1}, \mathrm{P}_{2}, \mathrm{~F}_{1}, \mathrm{~F}_{2}, \mathrm{BC}_{1} \mathrm{~F}_{1} \mathrm{P}_{1}\right.$ $\left(\mathrm{B}_{1}\right)$ and $\left.\mathrm{BC}_{1} \mathrm{~F}_{1} \mathrm{P}_{2} \quad\left(\mathrm{~B}_{2}\right)\right)$ were generated phenotypically distinguish parents (Table 1 and Fig. 1), evaluated during 2016-17 at College of Agriculture, UAS, Dharwad. Geographically, field is located at $15^{\circ} 29^{\prime} 46.8^{\prime \prime N o r t h}$ latitude and 74'59'11.3" East longitude at an altitude of $678 \mathrm{~m}$ above mean sea level with an average rainfall of $722.73 \mathrm{~mm}$. The soil type of the experimental block was vertisol with a $\mathrm{pH}$ in the range of 7 to 7.5 and plots were homogeneous with respect to soil nutrient status. Before sowing, seeds were treated with Imidacloprid to protect the crop from the incidence of sucking pests during early growth stage. Seeds were hand dibbled in rows of $8 \mathrm{~m}$ length with spacing of $90 \mathrm{~cm}$ between rows and $40 \mathrm{~cm}$ between plants. The average rainfall for the year 2016 was $563.1 \mathrm{~mm}$. During the critical period of crop growth (June to October-2016) the rainfall was $451.1 \mathrm{~mm}$, which was 24.9 per cent lesser than the 25 year's average rainfall $(600.68 \mathrm{~mm})$. However four protective irrigations were given at critical crop growth stages to raise good crop stand in order to realize its potential expression. Recommended package of practices for irrigated conditions of the south zone was followed to raise good crop.

The seed cotton yield and its components traits were recorded from the individual plants of $F_{2}$ (1329), $B_{1} \quad$ (174) and $B_{2}$ (191) populations, and average of 10 randomly selected plants of parents (Suvin and BCS 2318-7) and $F_{1}$. Plant height was measured in centimeters from the base of the plant to the apex of the plant at boll opening stage. Branches on main stem which were lateral and axillary in position with vertical growth in acropetal succession counted as a monopodia per plant. Branches which are extra axillary in position and normally horizontal with zigzag 
pattern of fruiting points were taken as sympodia. After picking of kapas, number of harvested bolls per plant was counted. Total seed cotton yield harvested from all pickings from each plant were weighed by electronic weighing balance and expressed in gram per plant.

Boll weight was calculated by dividing seed cotton yield per plant by number of bolls per plant and expressed in grams. The harvested kapas were ginned by ginning machine and determined the ginning out turn and expressed in percentage. This was calculated by using the formula.

$$
\begin{array}{r}
\text { Weight of lint }(\mathrm{g}) \\
\text { Weight of seed cotton }(\mathrm{g})
\end{array}
$$

Seed index was determined by weight of 100 seeds expressed in grams. Lint index is the weight of lint obtained from 100 seeds and expressed in grams. This was calculated by using the formula.

Seed index $\times$ Ginning outturn

$$
\begin{aligned}
& \text { Lint index = ------------------------------------- } \\
& \text { 100-Ginning out turn (\%) }
\end{aligned}
$$

Fibre quality traits of parents, $\mathrm{F}_{1}, 680 \mathrm{~F}_{2}, 174$ $\mathrm{B}_{1}$ and $191 \mathrm{~B}_{2}$ plants were analyzed with high volume index (HVI) instrument at Sujwal Bio Fuel, Belure Industrial area, Dharwad (India).

\section{Results and Discussion}

In genetics, transgressive segregation is the formation of extreme (transgressive) phenotypes, observed in segregating populations as compared to parents. There are many causes for transgressive segregation that are recombination of additive alleles, high mutation rate, reduced developmental stability and epistasis. Recombination results in new pairs of alleles at two or more loci and this new alleles pair can give rise to new/extreme phenotypes. When mutation rates are high, it is more probable that a mutation will occur and cause an extreme phenotypic change. Developmental stability refers to the capability of a genotype to go through a constant development of a phenotype in a certain environmental setting. If there is a disturbance due to genetic or environmental factors, the genotype will be more sensitive to phenotypic changes. Epistasis is the event when one allele at a locus influences an allele at another locus to express its product. All these causes lead to the appearance of these extreme phenotypes and creates a hybrid species that will deviate from the parent species niche.

The observed transgressive segregation for different traits in cotton segregating populations $\left(\mathrm{F}_{2}, \mathrm{~B}_{1}\right.$ and $\left.\mathrm{B}_{2}\right)$ of the present study presented in Table 2 (Fig. 2). Improvement of seed cotton yield is very much important in Gossypium barbadense, percentage transgressive segregants over superior parent BCS 23-18-7 in different segregation population is depicted in Table 3 (Fig. 3). Similarly Basamma et al., (2010) observed different frequency of transgressive segregants in 5 different $F_{2}$ populations of desi cotton.

The Observed trends for per cent transgressive segregation with decreasing order for seed cotton yield (SCY), number of sympodia per plant (NSP), number of bolls per plant (NBP), boll weight $(\mathrm{BW})$, ginning outturn (GOT) and lint index (LI) in $\mathrm{F}_{2}, \mathrm{~B}_{1}$ and $\mathrm{B}_{2}$ population is mentioned below.

With respect to SCY and NBP higher number of segregants observed in $\mathrm{B}_{2}$ population, for LI and NSP higher number of segregants observed in $\mathrm{B}_{1}$ population it may be due to BCS 23-18-7 superior for SCY and NBP, and Suvin superior for LI and NSP. 
Fig.1 (a) Suvin, (b) BCS 23-18-7 and (c) fibre length (Hallo length) difference

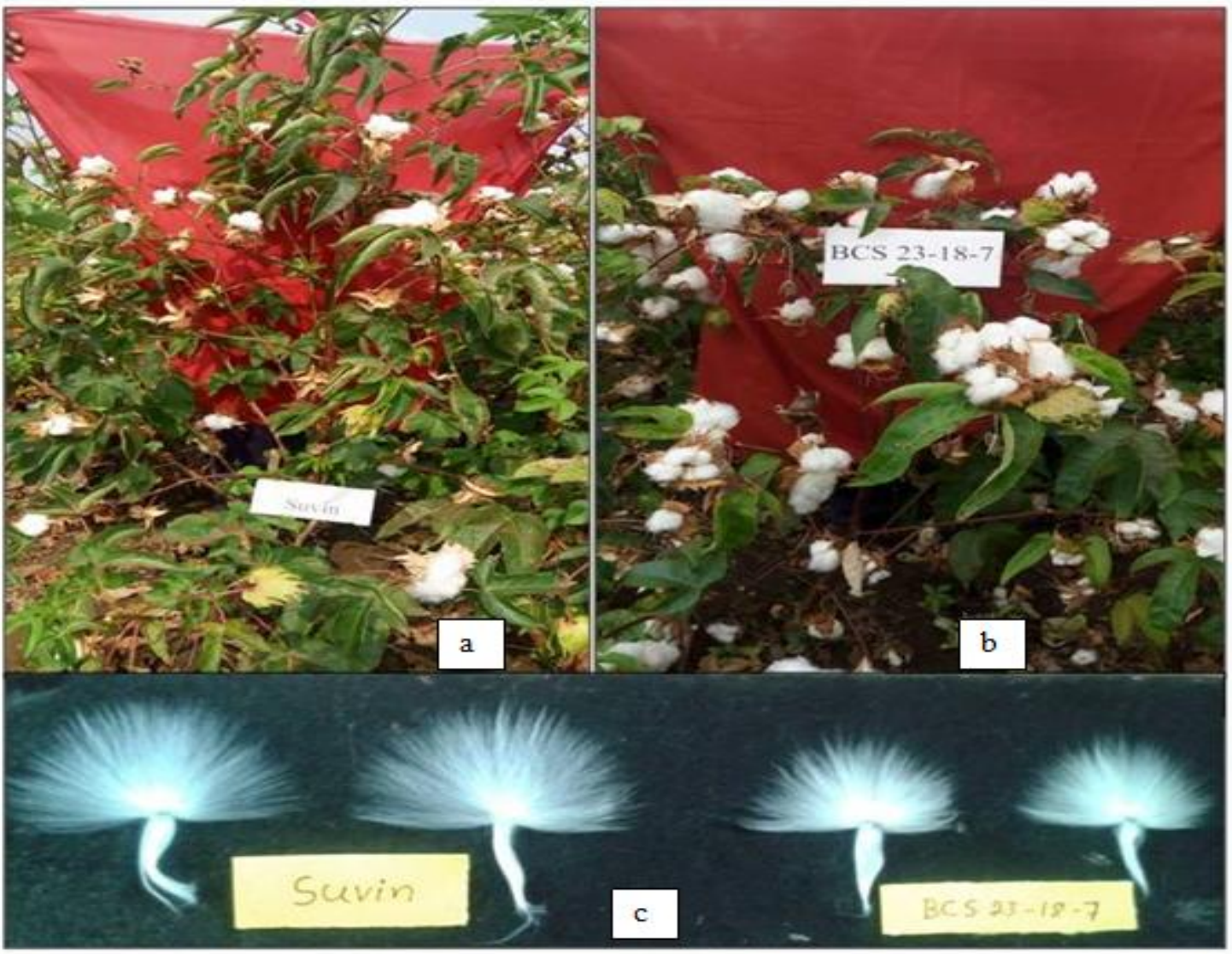

Fig.2 Number of transgressive segregants expressed in percentage

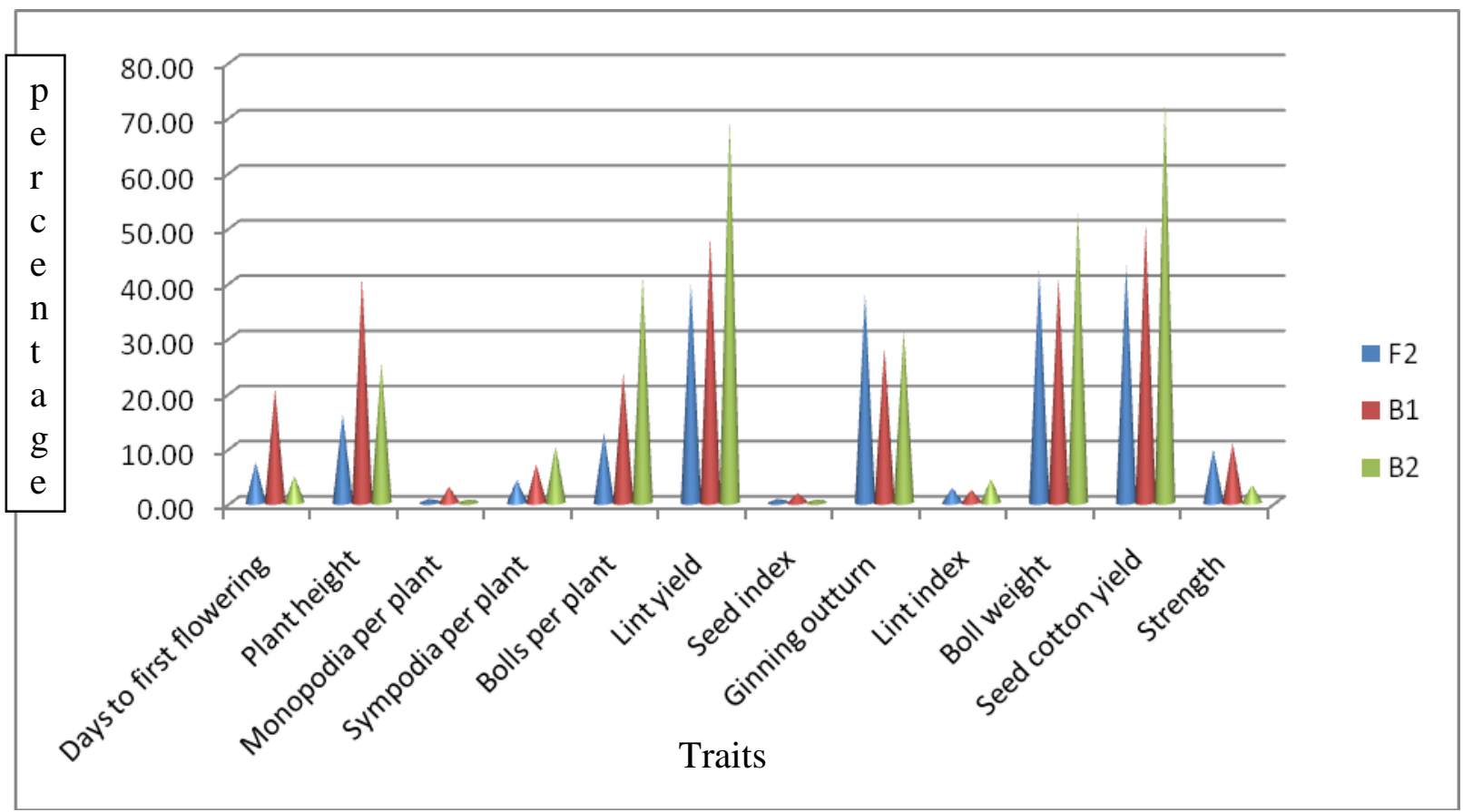


Fig.3 Number of transgressive segregants for seed cotton yield expressed in percentage over BCS 23-18-7 $\left(\mathrm{P}_{2}\right)$

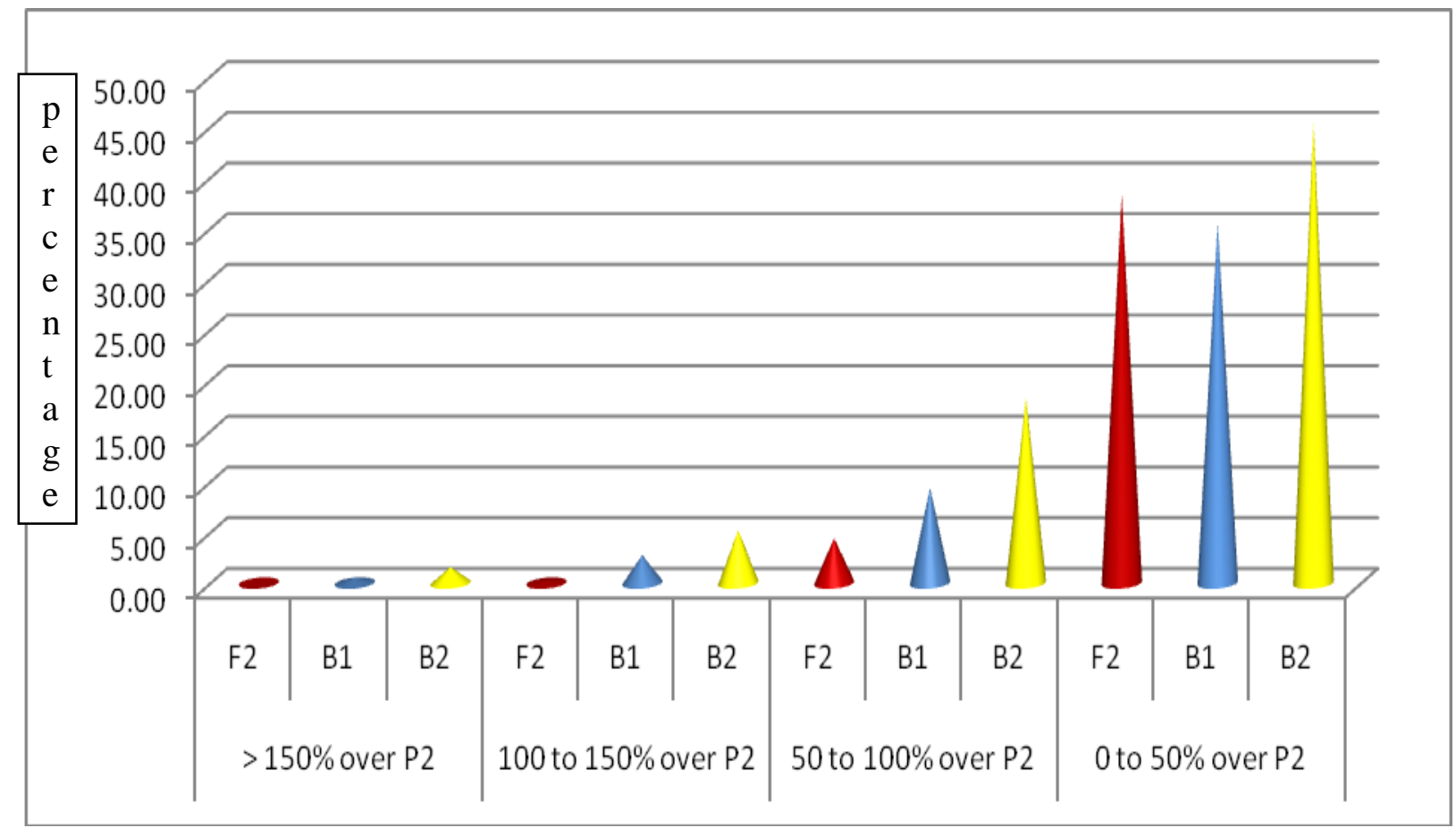

The Observed trends for per cent transgressive segregation with decreasing order

\begin{tabular}{|c|c|c|c|c|c|c|}
\hline SCY & NSP & NBP & BW & GOT & LI & $\begin{array}{c}\text { Decreasing } \\
\text { order }\end{array}$ \\
\hline $\mathrm{B}_{2}$ & $\mathrm{~B}_{1}$ & $\mathrm{~B}_{2}$ & $\mathrm{~B}_{2}$ & $\mathrm{~F}_{2}$ & $\mathrm{~B}_{1}$ & \\
\hline $\mathrm{B}_{1}$ & $\mathrm{~F}_{2}$ & $\mathrm{~B}_{1}$ & $\mathrm{~F}_{2}$ & $\mathrm{~B}_{2}$ & $\mathrm{~B}_{2}$ \\
\hline $\mathrm{F}_{2}$ & $\mathrm{~B}_{2}$ & $\mathrm{~F}_{2}$ & $\mathrm{~B}_{1}$ & $\mathrm{~B}_{1}$ & $\mathrm{~F}_{2}$ \\
\hline
\end{tabular}

Table.1 Characteristics of parents

\begin{tabular}{|c|c|c|c|c|c|c|}
\hline Varieties & $\begin{array}{c}\text { Fibre } \\
\text { length } \\
\text { (mm) }\end{array}$ & $\begin{array}{c}\text { Fibre } \\
\text { fineness } \\
\text { (Mic) }\end{array}$ & $\begin{array}{c}\text { Fibre } \\
\text { strength } \\
\text { (g/tex) }\end{array}$ & $\begin{array}{c}\text { Seed cotton } \\
\text { yield } \\
\text { (g/plant) }\end{array}$ & $\begin{array}{c}\text { Days to } \\
\text { first } \\
\text { flowering }\end{array}$ & $\begin{array}{c}\text { Plant } \\
\text { height } \\
(\mathrm{cm})\end{array}$ \\
\hline Suvin & $35-38$ & $3.2-3.3$ & 33.5 & $90-100$ & 79 & $14-150$ \\
\hline BCS 23- & $28-29$ & $3.4-3.5$ & 30 & $115-120$ & 68 & $115-120$ \\
\hline $\mathbf{1 8 - 7}$ & & & & & & \\
\hline
\end{tabular}


Int.J.Curr.Microbiol.App.Sci (2018) 7(1): 1040-1051

Table.2 Number of transgressive segregants expressed in percentage

\begin{tabular}{|c|c|c|c|c|c|c|c|}
\hline \multirow[t]{2}{*}{ Traits } & \multirow{2}{*}{$\begin{array}{c}\text { Over } P_{1} \\
\text { or } P_{2}\end{array}$} & \multicolumn{2}{|c|}{$F_{2}$} & \multicolumn{2}{|c|}{$\mathbf{B}_{1}$} & \multicolumn{2}{|c|}{$\mathbf{B}_{2}$} \\
\hline & & Number & $\begin{array}{l}\text { Per } \\
\text { cent }\end{array}$ & Number & Per cent & Number & $\begin{array}{l}\text { Per } \\
\text { cent }\end{array}$ \\
\hline \multicolumn{8}{|c|}{ Positive side } \\
\hline Days to first flowering & $\mathrm{P}_{1}(78)$ & 97 & 7.30 & 36 & 20.69 & 9 & 4.86 \\
\hline Plant height (cm) & $\begin{array}{c}\mathrm{P}_{1} \\
(147.21)\end{array}$ & 213 & 16.03 & 71 & 40.80 & 47 & 25.41 \\
\hline Monopodia per plant & P1 (5) & 9 & 0.68 & 5 & 2.87 & 1 & 0.54 \\
\hline Sympodia per plant & $\mathrm{P}_{1}(17)$ & 55 & 4.14 & 12 & 6.90 & 19 & 10.27 \\
\hline Bolls per plant & $\mathrm{P}_{2}(37)$ & 168 & 12.64 & 41 & 23.56 & 76 & 41.08 \\
\hline Lint yield (g) & $\mathrm{P}_{2}(39)$ & 530 & 39.88 & 84 & 48.28 & 129 & 69.73 \\
\hline Seed index (g) & $P_{1}(11.73)$ & 9 & 0.68 & 3 & 1.72 & 1 & 0.54 \\
\hline Ginning outturn (\%) & $\mathrm{P}_{2}(33.49)$ & 503 & 37.85 & 49 & 28.16 & 58 & 31.35 \\
\hline Lint index (g) & $\mathrm{P}_{1}(5.85)$ & 36 & 2.71 & 4 & 2.30 & 8 & 4.32 \\
\hline Boll weight (g) & $\mathrm{P}_{1}(3.85)$ & 559 & 42.06 & 71 & 40.80 & 98 & 52.97 \\
\hline $\begin{array}{l}\text { Seed cotton yield per } \\
\text { plant }(\mathrm{g})\end{array}$ & $\mathrm{P}_{2}(116.9)$ & 573 & 43.12 & 88 & 50.57 & 134 & 72.43 \\
\hline Fibre strength (g/tex) & $\mathrm{P}_{1}(33.47)$ & 64 & 9.52 & 19 & 10.92 & 6 & 3.24 \\
\hline \multicolumn{8}{|c|}{ Negative side } \\
\hline Days to first flowering & $\mathrm{P}_{2}(68)$ & 85 & 6.40 & 7 & 4.02 & 43 & 23.24 \\
\hline Monopodia & $\mathrm{P}_{2}(1.72)$ & 242 & 18.21 & 12 & 6.90 & 62 & 33.51 \\
\hline
\end{tabular}

$\mathrm{P}_{1}$ - Suvin and $\mathrm{P}_{2}-\mathrm{BCS} 23-18-7$

Table.3 Number of transgressive segregants for seed cotton yield expressed in percentage over BCS 23-18-7 $\left(\mathrm{P}_{2}\right)$

\begin{tabular}{|c|c|c|c|c|}
\hline Classes & Total Number & Populations & Number & Percentage \\
\hline \multirow{3}{*}{$>150 \%$ over $P_{2}$} & \multirow[t]{3}{*}{4} & $F_{2}$ & 0 & 0.00 \\
\hline & & $\mathrm{B}_{1}$ & 1 & 0.31 \\
\hline & & $\mathrm{B}_{2}$ & 3 & 0.82 \\
\hline \multirow{3}{*}{$\begin{array}{c}100 \text { to } 150 \% \text { over } \\
P_{2}\end{array}$} & \multirow[t]{3}{*}{19} & $\mathrm{~F}_{2}$ & 4 & 0.02 \\
\hline & & $\mathrm{B}_{1}$ & 5 & 1.54 \\
\hline & & $\mathrm{B}_{2}$ & 10 & 2.74 \\
\hline \multirow[t]{3}{*}{50 to $100 \%$ over $P_{2}$} & \multirow[t]{3}{*}{111} & $\mathrm{~F}_{2}$ & 59 & 0.33 \\
\hline & & $\mathrm{B}_{1}$ & 17 & 5.25 \\
\hline & & $\mathrm{B}_{2}$ & 35 & 9.59 \\
\hline \multirow[t]{3}{*}{0 to $50 \%$ over $P_{2}$} & \multirow[t]{3}{*}{661} & $\mathrm{~F}_{2}$ & 510 & 2.89 \\
\hline & & $\mathrm{B}_{1}$ & 64 & 19.75 \\
\hline & & $\mathrm{B}_{2}$ & 87 & 23.85 \\
\hline
\end{tabular}


Table.4 Top 20 transgressive segregants for seed cotton yield

\begin{tabular}{|c|c|c|c|c|c|c|c|c|c|c|c|c|c|c|}
\hline $\begin{array}{l}\text { Sl. } \\
\text { No. }\end{array}$ & PI No. & DF & $\begin{array}{c}\text { Pl. Ht } \\
\text { (cm) }\end{array}$ & Mon & Sym & Bolls & $\begin{array}{c}\text { LY } \\
\text { (g/plant) }\end{array}$ & $\begin{array}{l}\text { SI } \\
(\mathrm{g})\end{array}$ & $\begin{array}{c}\text { GOT } \\
(\%)\end{array}$ & $\begin{array}{l}\text { LI } \\
(\mathrm{g})\end{array}$ & $\begin{array}{c}\text { boll wt } \\
\text { (g) }\end{array}$ & $\begin{array}{c}\text { SCY } \\
\text { (g/plant) }\end{array}$ & $\begin{array}{c}\% \text { over } \\
\mathbf{P}_{2}\end{array}$ & $\%$ over $\mathbf{F}_{1}$ \\
\hline 1 & E126 & 72 & 112 & 4 & 26 & 54 & 110.50 & 10.48 & 35.65 & 5.8 & 5.74 & 315.00 & 169.46 & 98.86 \\
\hline 2 & D166 & 67 & 138 & 5 & 15 & 58 & 90.00 & 10.29 & 28.71 & 4.14 & 5.41 & 310.50 & 165.61 & 96.02 \\
\hline 3 & E135 & 70 & 130 & 3 & 13 & 52 & 101.00 & 10.01 & 33.17 & 4.97 & 5.86 & 304.50 & 160.48 & 92.23 \\
\hline 4 & E191 & 74 & 115 & 3 & 11 & 52 & 96.00 & 10.25 & 31.74 & 4.77 & 5.82 & 302.50 & 158.77 & 90.97 \\
\hline 5 & D149 & 71 & 149 & 4 & 13 & 49 & 92.00 & 10.59 & 32.45 & 5.09 & 5.79 & 283.50 & 142.51 & 78.98 \\
\hline 6 & E153 & 79 & 106 & 3 & 15 & 52 & 91.00 & 9.99 & 32.97 & 4.91 & 5.31 & 276.00 & 136.10 & 74.24 \\
\hline 7 & D69 & 71 & 132 & 4 & 14 & 50 & 76.00 & 10.06 & 28.04 & 3.92 & 5.42 & 271.00 & 131.82 & 71.09 \\
\hline 8 & E065 & 66 & 157 & 3 & 19 & 44 & 87.00 & 8.58 & 33.53 & 4.33 & 5.9 & 259.50 & 121.98 & 63.83 \\
\hline 9 & F443 & 72 & 120 & 4 & 8 & 49 & 80.50 & 9.77 & 31.08 & 4.41 & 5.29 & 259.00 & 121.56 & 63.51 \\
\hline 10 & E176 & 70 & 148 & 3 & 19 & 52 & 85.00 & 9.36 & 33.8 & 4.78 & 4.84 & 251.50 & 115.14 & 58.78 \\
\hline 11 & F298 & 65 & 200 & 4 & 17 & 42 & 78.50 & 10.08 & 31.27 & 4.59 & 5.98 & 251.00 & 114.71 & 58.46 \\
\hline 12 & D151 & 73 & 145 & 4 & 16 & 43 & 89.00 & 8.95 & 35.53 & 4.93 & 5.83 & 250.50 & 114.29 & 58.14 \\
\hline 13 & E112 & 70 & 135 & 3 & 11 & 45 & 85.50 & 9.41 & 34.13 & 4.88 & 5.57 & 250.50 & 114.29 & 58.14 \\
\hline 14 & E058 & 73 & 140 & 2 & 17 & 47 & 80.50 & 11.08 & 32.39 & 5.31 & 5.29 & 248.50 & 112.57 & 56.88 \\
\hline 15 & D178 & 75 & 135 & 3 & 12 & 48 & 81.50 & 9.56 & 33.2 & 4.75 & 5.11 & 245.50 & 110.01 & 54.99 \\
\hline 16 & F953 & 74 & 116 & 3 & 8 & 51 & 89.50 & 8.84 & 36.91 & 5.17 & 4.75 & 242.50 & 107.44 & 53.09 \\
\hline 17 & E027 & 69 & 130 & 3 & 14 & 44 & 82.50 & 9.44 & 34.09 & 4.88 & 5.5 & 242.00 & 107.01 & 52.78 \\
\hline 18 & E028 & 63 & 130 & 2 & 15 & 41 & 79.50 & 9.45 & 32.99 & 4.65 & 5.88 & 241.00 & 106.16 & 52.15 \\
\hline 19 & D32 & 72 & 146 & 4 & 16 & 43 & 84.50 & 8.78 & 35.36 & 4.8 & 5.56 & 239.00 & 104.45 & 50.88 \\
\hline 20 & F612 & 74 & 135 & 5 & 14 & 42 & 78.00 & 9.46 & 32.7 & 4.6 & 5.68 & 238.50 & 104.02 & 50.57 \\
\hline$\overline{\mathbf{P}_{1}}$ & Suvin & 78.84 & 147.21 & 4.79 & 17.37 & 25.74 & 32.31 & 11.73 & 33.26 & 5.85 & 3.85 & 97.18 & - & \\
\hline \multirow[t]{2}{*}{$\mathbf{P}_{2}$} & $\begin{array}{c}\text { BCS } \\
23-18-7\end{array}$ & 68.22 & 118.06 & 1.72 & 16.17 & 37.17 & 29.46 & 8.95 & 33.49 & 4.5 & 3.14 & 116.9 & - & \\
\hline & $\mathrm{F}_{1}$ & 73.64 & 126.97 & 3.69 & 15.61 & 39.72 & 53.32 & 9.55 & 33.75 & 4.86 & 3.97 & 158.04 & - & \\
\hline
\end{tabular}

DF- Days to first flowering SI- Seed Index

F- F 2 population
Pl.Ht- Plant height

GOT- Ginning outturn

D- $B_{1}$ population
Mon- Monopodia per plant

Sym-Sympodia per plant

SCY - Seed cotton yield per plant

LI- Lint Index
E- $\mathbf{B}_{2}$ population

LY- Lint Yield per plant 
Table.5 Transgressive segregants with 5 per cent advantage ( $<65$ days) to first flowering with 50 per cent (177 g) yield advantage over BCS-23-18-7

\begin{tabular}{|c|c|c|c|c|c|c|c|c|c|c|c|c|c|c|}
\hline Sl. No. & $\begin{array}{c}\text { Pl } \\
\text { No. }\end{array}$ & DF & $\begin{array}{l}\text { Pl. Ht } \\
(\mathrm{cm})\end{array}$ & Mon & Sym & Bolls & $\begin{array}{c}\text { LY } \\
\text { (g/plant) }\end{array}$ & $\begin{array}{l}\text { SI } \\
(\mathrm{g})\end{array}$ & $\begin{array}{c}\text { GOT } \\
(\%)\end{array}$ & $\begin{array}{l}\text { LI } \\
(\mathrm{g})\end{array}$ & $\begin{array}{c}\text { boll } \\
\text { wt } \\
(\mathrm{g})\end{array}$ & $\begin{array}{c}\text { SCY } \\
\text { (g/plant) }\end{array}$ & $\begin{array}{c}\% \text { over } \\
\mathbf{P}_{2}\end{array}$ & $\begin{array}{c}\% \\
\text { over } \\
\text { F }_{1}\end{array}$ \\
\hline 1 & E028 & 63 & 130 & 2 & 15 & 41 & 79.50 & 9.45 & 32.99 & 4.65 & 5.88 & 241.00 & 106.16 & 52.15 \\
\hline 2 & E179 & 62 & 122 & 2 & 16 & 48 & 77.00 & 9.15 & 33.7 & 4.65 & 4.76 & 228.50 & 95.47 & 44.26 \\
\hline 3 & E015 & 63 & 125 & 3 & 16 & 40 & 72.50 & 8.91 & 34.04 & 4.60 & 5.33 & 213.00 & 82.21 & 34.47 \\
\hline 4 & E057 & 64 & 145 & 1 & 18 & 44 & 61.50 & 11.44 & 30.15 & 4.94 & 4.64 & 204.00 & 74.51 & 28.79 \\
\hline 5 & E105 & 63 & 145 & 2 & 15 & 44 & 64.00 & 9.63 & 32.65 & 4.67 & 4.45 & 196.00 & 67.66 & 23.74 \\
\hline 6 & E014 & 64 & 135 & 3 & 14 & 46 & 65.50 & 8.84 & 34.29 & 4.61 & 4.15 & 191.00 & 63.39 & 20.58 \\
\hline $\mathbf{P}_{1}$ & Suvin & 78.84 & 147.21 & 4.79 & 17.37 & 25.74 & 32.31 & 11.73 & 33.26 & 5.85 & 3.85 & 97.18 & - & \\
\hline $\mathbf{P}_{2}$ & $\begin{array}{c}\text { BCS } \\
23- \\
18-7\end{array}$ & 68.22 & 118.06 & 1.72 & 16.17 & 37.17 & 29.46 & 8.95 & 33.49 & 4.5 & 3.14 & 116.90 & - & \\
\hline & $\mathrm{F}_{1}$ & 73.64 & 126.97 & 3.69 & 15.61 & 39.72 & 53.32 & 9.55 & 33.75 & 4.86 & 3.97 & 158.04 & - & \\
\hline
\end{tabular}

\begin{tabular}{|c|c|c|c|c|}
\hline $\begin{array}{l}\text { DF- Days to first } \\
\text { flowering }\end{array}$ & Pl.Ht- Plant height & $\begin{array}{l}\text { Mon- Monopodia per } \\
\text { plant }\end{array}$ & $\begin{array}{l}\text { Sym- Sympodia per } \\
\text { plant }\end{array}$ & $\begin{array}{l}\text { LY- Lint Yield per } \\
\text { plant }\end{array}$ \\
\hline SI- Seed Index & Ginning & LI- Lint Index & \multicolumn{2}{|c|}{ SCY-Seed cotton yield per plant } \\
\hline F- $F_{2}$ population & D- $B_{1}$ population & E- $B_{2}$ population & & \\
\hline
\end{tabular}


Table.6 Transgressive segregants for more than 50 per cent yield advantage over BCS 23-18-7 (116.90 g) with lower monopodia

\begin{tabular}{|c|c|c|c|c|c|c|c|c|c|c|c|c|c|c|}
\hline Sl. No. & $\begin{array}{c}\text { Pl } \\
\text { No. }\end{array}$ & DF & $\begin{array}{l}\text { Pl. Ht } \\
(\mathrm{cm})\end{array}$ & Mon & Sym & Bolls & $\begin{array}{c}\text { LY } \\
\text { (g/plant) }\end{array}$ & $\begin{array}{l}\text { SI } \\
(\mathrm{g})\end{array}$ & $\begin{array}{c}\text { GOT } \\
(\%)\end{array}$ & $\begin{array}{l}\text { LI } \\
(\mathrm{g})\end{array}$ & $\begin{array}{c}\text { boll } \\
\text { wt } \\
(\mathrm{g})\end{array}$ & $\begin{array}{c}\text { SCY } \\
\text { (g/plant) }\end{array}$ & $\begin{array}{c}\% \text { over } \\
\mathbf{P}_{2}\end{array}$ & $\begin{array}{c}\% \\
\text { over } \\
\text { F }_{1}\end{array}$ \\
\hline 1 & E127 & 68 & 150 & 1 & 12 & 43 & 77.00 & 11.33 & 32.84 & 5.54 & 5.45 & 234.50 & 100.60 & 48.04 \\
\hline 2 & E024 & 66 & 160 & 1 & 18 & 45 & 81.50 & 10.10 & 37.30 & 6.01 & 4.86 & 218.50 & 86.91 & 37.94 \\
\hline 3 & E094 & 66 & 150 & 0 & 16 & 43 & 74.50 & 8.99 & 34.61 & 4.76 & 5.01 & 215.23 & 84.11 & 35.88 \\
\hline 4 & E037 & 68 & 133 & 1 & 10 & 45 & 55.50 & 8.87 & 26.18 & 3.15 & 4.71 & 212.00 & 81.35 & 33.84 \\
\hline 5 & E057 & 64 & 145 & 1 & 18 & 44 & 61.50 & 11.44 & 30.15 & 4.94 & 4.64 & 204.00 & 74.51 & 28.79 \\
\hline 6 & E182 & 65 & 140 & 1 & 14 & 44 & 69.50 & 8.63 & 34.66 & 4.58 & 4.56 & 200.50 & 71.51 & 26.58 \\
\hline 7 & E055 & 73 & 150 & 1 & 13 & 42 & 63.00 & 11.25 & 31.58 & 5.19 & 4.75 & 199.50 & 70.66 & 25.95 \\
\hline 8 & F695 & 74 & 147 & 1 & 7 & 32 & 64.00 & 9.31 & 35.26 & 5.07 & 5.67 & 181.50 & 55.26 & 14.58 \\
\hline 9 & E154 & 74 & 132 & 1 & 13 & 41 & 63.00 & 8.61 & 35.00 & 4.64 & 4.39 & 180.01 & 53.99 & 13.64 \\
\hline 10 & F912 & 79 & 135 & 1 & 11 & 32 & 60.50 & 8.94 & 33.61 & 4.53 & 5.63 & 180.00 & 53.98 & 13.64 \\
\hline 11 & F883 & 72 & 125 & 1 & 6 & 29 & 60.00 & 8.97 & 33.80 & 4.58 & 6.01 & 177.50 & 51.84 & 12.06 \\
\hline $\mathbf{P}_{1}$ & Suvin & 78.84 & 147.21 & 4.79 & 17.37 & 25.74 & 32.31 & 11.73 & 33.26 & 5.85 & 3.85 & 97.18 & - & \\
\hline $\mathbf{P}_{2}$ & $\begin{array}{c}\text { BCS } \\
23- \\
18-7\end{array}$ & 68.22 & 118.06 & 1.72 & 16.17 & 37.17 & 29.46 & 8.95 & 33.49 & 4.5 & 3.14 & 116.9 & - & \\
\hline & $\mathrm{F}_{1}$ & 73.64 & 126.97 & 3.69 & 15.61 & 39.72 & 53.32 & 9.55 & 33.75 & 4.86 & 3.97 & 158.04 & - & \\
\hline
\end{tabular}

\begin{tabular}{|c|c|c|c|c|}
\hline $\begin{array}{l}\text { DF- Days to first } \\
\text { flowering }\end{array}$ & Pl.Ht- Plant height & $\begin{array}{l}\text { Mon- Monopodia per } \\
\text { plant }\end{array}$ & $\begin{array}{l}\text { Sym- Sympodia per } \\
\text { plant }\end{array}$ & $\begin{array}{l}\text { LY- Lint Yield per } \\
\text { plant }\end{array}$ \\
\hline SI- Seed Index & $\begin{array}{l}\text { GOT- Ginning } \\
\text { outturn }\end{array}$ & LI- Lint Index & \multicolumn{2}{|c|}{ SCY-Seed cotton yield per plant } \\
\hline F- $F_{2}$ population & D- $B_{1}$ population & E- $B_{2}$ population & & \\
\hline
\end{tabular}


Table.7 Transgressive segregants for more than 5 per cent GOT advantage over BCS 23-18-7 (33.49 \%) with 50 per cent yield advantage over BCS 23-18-7 (116.90 g)

\begin{tabular}{|c|c|c|c|c|c|c|c|c|c|c|c|c|c|c|}
\hline $\begin{array}{l}\text { Sl. } \\
\text { No. }\end{array}$ & PI No. & DF & $\begin{array}{l}\text { Pl. Ht } \\
(\mathrm{cm})\end{array}$ & Mon & Sym & Bolls & $\begin{array}{c}\text { LY } \\
\text { (g/plant) }\end{array}$ & $\begin{array}{l}\text { SI } \\
(\mathrm{g})\end{array}$ & $\begin{array}{c}\text { GOT } \\
(\%)\end{array}$ & $\begin{array}{l}\text { LI } \\
(\mathrm{g})\end{array}$ & $\begin{array}{c}\text { boll } \\
\text { wt } \\
(\mathrm{g})\end{array}$ & $\begin{array}{c}\text { SCY } \\
\text { (g/plant) }\end{array}$ & $\%$ over $\mathbf{P}_{2}$ & $\begin{array}{c}\% \\
\text { over } \\
\text { F }_{1}\end{array}$ \\
\hline 1 & F960 & 73 & 135 & 2 & 14 & 48 & 87 & 8.83 & 40.75 & 6.07 & 4.45 & 213.50 & 82.63 & 34.79 \\
\hline 3 & F953 & 74 & 116 & 3 & 8 & 51 & 89.5 & 8.84 & 36.91 & 5.17 & 4.75 & 242.50 & 107.44 & 53.09 \\
\hline 4 & E090 & 73 & 150 & 2 & 15 & 33 & 67 & 9.96 & 36.61 & 5.75 & 5.55 & 183.00 & 56.54 & 15.53 \\
\hline 5 & D113 & 80 & 132 & 4 & 12 & 35 & 68.5 & 9.86 & 35.96 & 5.54 & 5.44 & 190.50 & 62.96 & 20.27 \\
\hline 7 & E005 & 79 & 125 & 4 & 10 & 35 & 74 & 8.93 & 35.75 & 4.97 & 5.91 & 207.00 & 77.07 & 30.68 \\
\hline 8 & F327 & 68 & 130 & 3 & 9 & 43 & 65 & 10.00 & 35.71 & 5.56 & 4.23 & 182.00 & 55.69 & 14.90 \\
\hline 9 & E126 & 72 & 112 & 4 & 26 & 54 & 110.5 & 10.48 & 35.65 & 5.8 & 5.74 & 310.00 & 165.18 & 95.71 \\
\hline 10 & D151 & 73 & 145 & 4 & 16 & 43 & 89 & 8.95 & 35.53 & 4.93 & 5.83 & 250.50 & 114.29 & 58.14 \\
\hline 11 & F1262 & 72 & 135 & 3 & 9 & 35 & 68.5 & 7.98 & 35.49 & 4.39 & 5.51 & 193.00 & 65.10 & 21.84 \\
\hline 12 & D32 & 72 & 146 & 4 & 16 & 43 & 84.5 & 8.78 & 35.36 & 4.8 & 5.56 & 239.00 & 104.45 & 50.88 \\
\hline $\mathbf{P}_{1}$ & Suvin & 78.84 & 147.21 & 4.79 & 17.37 & 25.74 & 32.31 & 11.73 & 33.26 & 5.85 & 3.85 & 97.18 & - & \\
\hline $\mathbf{P}_{2}$ & $\begin{array}{c}\text { BCS 23- } \\
18-7\end{array}$ & 68.22 & 118.06 & 1.72 & 16.17 & 37.17 & 29.46 & 8.95 & 33.49 & 4.50 & 3.14 & 116.9 & - & \\
\hline & $\mathrm{F}_{1}$ & 73.64 & 126.97 & 3.69 & 15.61 & 39.72 & 53.32 & 9.55 & 33.75 & 4.86 & 3.97 & 158.04 & - & \\
\hline
\end{tabular}

\begin{tabular}{|c|c|c|c|c|}
\hline $\begin{array}{l}\text { DF- Days to first } \\
\text { flowering }\end{array}$ & Pl.Ht- Plant height & $\begin{array}{l}\text { Mon- Monopodia per } \\
\text { plant }\end{array}$ & $\begin{array}{l}\text { Sym- Sympodia per } \\
\text { plant }\end{array}$ & $\begin{array}{l}\text { LY- Lint Yield per } \\
\text { plant }\end{array}$ \\
\hline SI- Seed Index & $\begin{array}{l}\text { GOT- } \\
\text { outturn }\end{array}$ & LI- Lint Index & \multicolumn{2}{|c|}{ SCY-Seed cotton yield per plant } \\
\hline $\mathrm{F}-\mathrm{F}_{2}$ population & D- $B_{1}$ population & E- $B_{2}$ population & & \\
\hline
\end{tabular}


Table.8 Transgressive segregants for combination of high strength (> 3\% over Suvin) with 25 per cent yield advantage over BCS 23$18-7(116.90 \mathrm{~g})$

\begin{tabular}{|c|c|c|c|c|c|c|c|c|c|c|c|c|c|}
\hline $\begin{array}{l}\text { Sl. } \\
\text { No. }\end{array}$ & PI No. & $\begin{array}{l}\text { UHML } \\
(\mathrm{mm})\end{array}$ & $\begin{array}{c}\text { Strength } \\
\text { (g/tex) }\end{array}$ & 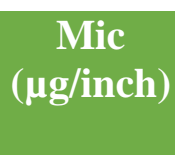 & $\begin{array}{l}\text { UR } \\
(\%)\end{array}$ & $\begin{array}{l}\text { MR } \\
(\%)\end{array}$ & $\begin{array}{l}\text { EL } \\
(\%)\end{array}$ & $\begin{array}{l}\text { SI } \\
(\mathbf{g})\end{array}$ & $\begin{array}{l}\text { LI } \\
(\mathbf{g})\end{array}$ & $\begin{array}{c}\text { GOT } \\
(\%)\end{array}$ & $\begin{array}{c}\text { SCY } \\
\text { (g/plant) }\end{array}$ & $\begin{array}{c}\% \\
\text { over } \\
\mathbf{P}_{2}\end{array}$ & $\begin{array}{c}\% \\
\text { over } \\
\mathbf{F}_{1}\end{array}$ \\
\hline 1 & F425 & 31.70 & 34.8 & 3.74 & 92.50 & 0.66 & 6.70 & 8.79 & 4.66 & 34.67 & 225.00 & 92.47 & 42.05 \\
\hline 2 & F179 & 34.00 & 38.3 & 3.65 & 92.60 & 0.64 & 6.80 & 9.70 & 3.93 & 28.84 & 215.00 & 83.92 & 35.73 \\
\hline 3 & D110 & 34.00 & 35.9 & 3.10 & 84.40 & 0.57 & 5.90 & 10.18 & 4.99 & 32.90 & 193.00 & 65.10 & 21.84 \\
\hline 4 & E102 & 29.50 & 34.1 & 3.65 & 34.80 & 0.66 & 5.20 & 8.68 & 4.54 & 34.32 & 185.00 & 58.25 & 16.79 \\
\hline 5 & F100 & 32.70 & 36.3 & 3.43 & 93.70 & 0.60 & 6.80 & 9.15 & 4.81 & 34.46 & 177.00 & 51.41 & 11.74 \\
\hline 6 & F236 & 32.60 & 36.4 & 3.51 & 93.10 & 0.62 & 6.70 & 11.95 & 5.64 & 32.08 & 159.00 & 36.01 & 0.38 \\
\hline 7 & F408 & 33.60 & 34.6 & 3.18 & 92.30 & 0.55 & 6.60 & 8.80 & 4.07 & 31.65 & 158.00 & 35.16 & -0.25 \\
\hline 8 & F220 & 31.30 & 34.0 & 3.47 & 93.10 & 0.61 & 6.70 & 11.50 & 6.20 & 35.03 & 157.00 & 34.30 & -0.88 \\
\hline 9 & D120 & 33.40 & 34.7 & 3.37 & 85.60 & 0.61 & 6.00 & 11.14 & 5.23 & 31.94 & 155.00 & 32.59 & -2.15 \\
\hline 10 & D24 & 32.60 & 34.8 & 3.65 & 82.20 & 0.66 & 5.30 & 9.37 & 4.66 & 33.22 & 152.00 & 30.03 & -4.04 \\
\hline 11 & F205 & 34.30 & 38.8 & 3.01 & 93.80 & 0.53 & 6.70 & 9.98 & 4.89 & 32.89 & 150.50 & 28.74 & -4.99 \\
\hline 12 & F422 & 33.70 & 35.8 & 3.94 & 92.70 & 0.71 & 6.50 & 10.23 & 6.18 & 37.67 & 150.00 & 28.31 & -5.30 \\
\hline 13 & F213 & 34.20 & 36.9 & 3.16 & 91.30 & 0.55 & 6.70 & 10.05 & 4.63 & 31.54 & 149.00 & 27.46 & -5.93 \\
\hline 14 & F544 & 30.90 & 34.1 & 3.50 & 79.90 & 0.63 & 5.00 & 10.56 & 5.39 & 33.78 & 148.00 & 26.60 & -6.57 \\
\hline $\mathbf{P}_{1}$ & Suvin & 35.85 & 33.47 & 3.36 & 85.28 & 0.61 & 6.22 & 11.73 & 5.85 & 33.26 & 97.18 & & \\
\hline$\overline{\mathbf{P}_{2}}$ & $\begin{array}{c}\text { BCS } \\
23-18- \\
7\end{array}$ & 28.32 & 31.55 & 3.71 & 82.65 & 0.68 & 5.03 & 8.95 & 4.5 & 33.49 & 116.9 & & \\
\hline & $\mathrm{F}_{1}$ & 32 & 32.37 & 3.43 & 85.34 & 0.63 & 5.97 & 9.55 & 4.86 & 33.75 & 158.04 & & \\
\hline
\end{tabular}

UHML-Upper half mean length

MR- Fibre maturity ratio

GOT-Ginning outturn

F- $F_{2}$ population
Mic- Micronaire value

EL- Fibre elongation

LI- Lint Index

D- $B_{1}$ population
UR- Fibre uniformity ratio

SI- Seed Index

SCY-Seed cotton yield per plant

E- B 2 population 
The top 20 seed cotton yield transgressive segregants selected from all three segregation generations $\left(F_{2}, B_{1}\right.$ and $\left.B_{2}\right)$, recorded yield advantage ranges from 104.02 to 169.46 per cent over BCS 23-18-7 (116.9 g) and their per se performance is shown in Table 4. Plant numbers $F, D$ and $E$ series indicated the $F_{2}, B_{1}$ and $B_{2}$ population respectively. Out of these twenty plants, 8 were from only $\mathrm{B}_{2}$ population, this may be due to back crossed parent BCS 23 18-7 having high yielding ability than other parent Suvin and these selected plants are valuable resource for improving yield in $G$. barbadense. Plant number E126 recorded $315 \mathrm{~g}$ per plant because this plant recorded higher boll number (54), boll weight $(5.74 \mathrm{~g})$, lint index $(5.8 \mathrm{~g})$ and seed index (10.48g). Six transgressive segregants (Table 5) recorded 5 per cent early flowering ( $<65$ days) with a minimum of 50 per cent yield advantage $(>177$ g) over BCS 23-18-7 and these selected plants will be helpful in improving early maturity with high yield in $G$ barbadense. Eleven plants (Table 6) were recorded 1 or less than 1 monopodia with more than 50 per cent $(177 \mathrm{~g})$ yield advantage and these selected will help to improve plants suitable for high density planting with high yield. There were fifteen plants showed transgressive segregation for the combination of seed cotton yield and GOT with 50 and 5 per cent advantage respectively over superior parent BCS 23-18-7 (Table 7), and these selected plants are the valuable resource in future for improving both yield and GOT simultaneously. Usually seed cotton yield negatively correlated with fibre quality traits, but in the present study 14 transgressive segregants were found in combination of 3 per cent $(34.5 \mathrm{~g} /$ tex $)$ fibre strength over Suvin with 25 per cent $(>145 \mathrm{~g})$ yield advantage over BCS 23-18-7 (Table 8) and these selected plants will be use full for improving simultaneously for strength with seed cotton yield. Since, these selected plants for different traits in the early segregation generation, they may not be stable, need to advance for 3 to 4 generation of following plant to progeny row with selection for desirable traits. Similarly Ramesh et al., (2017) recovered RILs with 21.72 to 44.72 per cent yield advantage RILs, 44.18 per cent GOT advantage derived from DCH 32, interspecific hybrid.

\section{References}

Anonymous, 2016. Cotton Industry India. https://www.ibef.org/exports/cottonindustry india.aspx.

Anonymous, 2017. Status paper of Indian cotton. http://www.nfsm.gov.in/Status Paper/Cotton Status2017.pdf. pp 21-31

Basamma K., Kajjidoni1 S. T., Salimath P. M. and Malagouda P., 2010. Productivity and fiber quality improvement through transgressive breeding in desi cotton. IJAS 6 (2): 609-614.

Katarki, B. H., 1971. Varalxmi, A high yielding hybrid cotton of quality. Indian Farming, 21(8): 35-36.

Ramesh M., Mohan K. N. V. and Katageri I. S., 2017. Identification of Superior Rils for Fibre Quality, Seed Cotton Yield and Its Component Traits. Int. J. Pure App. Biosci. 5 (3): 130-137.

Wendel J. F., Small R. L., Cronn R. C. and Brubaker C. L., Genes, jeans and genomes: reconstructing the history of cotton, in : van Raamsdonk L.W.D., Den Nijs J. C. M., 1999. Plant evolution of man-made habitats- Proceedings of $\mathrm{VII}^{\text {th }}$ international symposium of the international organization of plant biosystematists, Hugo de Vries Laboratory, Amsterdam, Netherlands.

\section{How to cite this article:}

Mohan Kumar, N.V., I.S. Katageri, G. Sunil Kumar and Anjan Gowda, S. 2018. Transgressive Segregation for Fibre Quality, Seed Cotton Yield and Its Component Traits in Gossypium barbadense L. Cotton. Int.J.Curr.Microbiol.App.Sci. 7(01): 1040-1051. doi: https://doi.org/10.20546/ijcmas.2018.701.125 